\title{
Asymmetric larval competition in the parasitoid wasp Nasonia vitripennis: a role in sex allocation?
}

\author{
Edward M. Sykes • Tabitha M. Innocent • Ido Pen • \\ David M. Shuker • Stuart A. West
}

Published online: 18 December 2007

(C) Springer-Verlag 2007

\section{Erratum to: Behav Ecol Sociobiol (2007) 61:1751-1758 DOI 10.1007/s00265-007-0407-1}

The original publication did not include the appendix to the article. The missing material is supplied here.

\section{Appendix}

Here we extend a standard model of sex ratio evolution under local mate competition (LMC) to take into account effects of clutch size and sex ratio on female fecundity or longevity, as we have observed in our experiments. We will then parameterize the model with empirical estimates of these relationships to generate quantitative predictions of sex ratios.

The model is built around the standard life cycle of $N$ mated females breeding in a patch. The mated females produce offspring that mate randomly among themselves within the patch, followed by dispersal of the newly mated females to random patches.

We use the direct fitness approach as developed by Taylor and Frank (1996) and Frank (1998), as we have

The online version of the original article can be found at http://dx.doi. org/10.1007/s00265-007-0407-1.

E. M. Sykes $(\bowtie) \cdot$ T. M. Innocent $\cdot$ D. M. Shuker $\cdot$ S. A. West Institute of Evolutionary Biology, School of Biological Sciences, University of Edinburgh,

West Mains Road,

Edinburgh, Scotland

e-mail: Ed.Sykes@ed.ac.uk

I. Pen

Theoretical Biology Group,

Centre for Ecological and Evolutionary Studies,

University of Groningen,

P.O. Box 14, 9750 AA Haren, The Netherlands done in previous papers on Nasonia sex ratios (Reece et al. 2004, Shuker et al. 2005).

A mother's total fitness is given by

$W=c_{f} W_{f}+c_{m} W_{m}$

where $W_{i}$ is her fitness through sex- $i$ offspring $(i=m, f)$ and $c_{i}$ is the class reproductive value of sex $i$. For haplodiploids, the reproductive value of females is twice that of males $\left(c_{f}=\right.$ $2 c_{m}$ ), which is another way of saying that a random gene from a population in the far future is twice as likely to be present today in a female as in a male (Taylor 1988). A focal female with sex ratio $x$ (proportion sons) and clutch size $k$ has fitness through daughters proportional to

$W_{f}=(1-x) F$

where $F=F(\bar{x}, k)$ is a daughter's expected reproductive success, which may depend on the average sex ratio $\bar{x}$ produced by the females in the patch and their clutch size $k$. We assume that $k$ is fixed, not being under selection, unlike in the models of Godfray (1986), where simultaneous selection on sex ratio and clutch size was studied. We will consider two extreme situations, one where $F$ is estimated by a female's fecundity and one where $F$ is estimated by her life expectancy. However, it turns out that the numerical predictions for both scenarios are virtually identical, and we will therefore show only the results for fecundity.

The focal female's fitness through sons is given by

$W_{m}=x \frac{1-\bar{x}}{\bar{x}} F$

The ratio $(1-\bar{x}) / \bar{x}$ is the expected number of mates per son and $F=F(\bar{x}, k)$ is theexpected reproductive success of those mates. 
The direction and strength of selection on the sex ratio is given by the selection gradient

$$
\begin{aligned}
\frac{\mathrm{d} W}{\mathrm{~d} x}= & r_{f} c_{f} \frac{\partial W_{f}}{\partial x}+R_{f} c_{f} \frac{\partial W_{f}}{\partial \bar{x}}+r_{m} c_{m} \frac{\partial W_{m}}{\partial x} \\
& +R_{m} c_{m} \frac{\partial W_{m}}{\partial \bar{x}}
\end{aligned}
$$

where $r_{i}$ is the relatedness of sex- $i$ offspring to the focal female and $R_{i}$ the relatedness to the focal female of random sex- $i$ offspring born in the same patch. The relatedness coefficients can be calculated from standard population genetic recursions (Taylor 1988, Reece et al. 2004). For haplodiploids with random dispersal of mated females, the results are

$r_{f}=1 /(2-1 / N), R_{f}=r_{f} / N, r_{m}=1, R_{m}=r_{m} / N$.

Working out the partial derivatives gives the selection gradient

$$
\begin{aligned}
\frac{\mathrm{d} W}{\mathrm{~d} x}= & -r_{f} c_{f} F+R_{f} c_{f}(1-\widehat{x}) F_{x}+r_{m} c_{m} \frac{1-\widehat{x}}{\widehat{x}} F \\
& -R_{m} c_{m}\left[\frac{F}{\widehat{x}}-(1-\widehat{x}) F_{x}\right]
\end{aligned}
$$

where all expressions on the right are evaluated at $x=\bar{x}=\widehat{x} . F_{x}$ represents the partial derivative of $F$ with respect to the average patch sex ratio $\bar{x}$, again evaluated at $x=\bar{x}=\widehat{x}$.

Female reproductive success $F$ as a function of body size $y$ was estimated with a linear model of the form

$F=\beta_{0}+\beta_{1} y$.

Body size $y$, in turn, was estimated as a function of sex ratio $x$ and clutch size $k$ by a model of the form

$y=\gamma_{0}+\gamma_{1} k+\gamma_{2} \arcsin (\sqrt{x})+\gamma_{3} k \cdot \arcsin (\sqrt{x})$.

Plugging this relationship into (A7) and taking the derivative with respect to $x$ yields the following estimate for $F_{x}$ :

$F_{x}=\frac{\beta_{1}\left(\gamma_{2}+\gamma_{3} k\right)}{2 \sqrt{x(1-x)}}$.

Predicted sex ratios were calculated numerically with Maple 9.5 as the $\widehat{x}$ roots of the right-hand side of (A6) after plugging in (A7) and (A9) and estimates of the various parameters. Figure 5 shows the standard predicted sex ratio $N(2 N-1) / N(4 N-1)$ (Hamilton 1979) and the predicted sex ratios from our model for Nasonia vitripennis (parameter estimates from table 2), assuming a total number of competitors of $k=70$. For smaller clutch sizes, the deviation from the standard model is even smaller. Figure 6 shows the very similar predictions for Bracon hebetor (data in the figure legend). 